\title{
SpécifiCITéS
}

La revue des terrains sensibles.

\section{DE LA RECHERCHE QUALITATIVE A LA RECHERCHE SENSIBLE}

La recherche qualitative est aujourd'hui reconnue comme une démarche compréhensive et flexible qui permet d'étudier les phénomènes sociaux en profondeur (Singelton \& Straits, 1999). Elle est caractérisée par une grande diversité (Denzin, 2010), néanmoins certains éléments transversaux sont communs à l'ensemble des approches qualitatives. C'est notamment le cas de la sensibilité qui semble être au cœur de la démarche qualitative. Certains évoquent une sensibilité pragmatique en considérant qu'une des qualités essentielles du chercheur qualitatif réside dans sa capacité à réagir en adéquation avec le terrain, la sensibilité est alors associée à une forme de sagesse (Cassell, Bishop, Symon, Johnson, \& Buehring, 2009). Pour d'autres, la posture sensible réside dans la capacité du chercheur à se saisir des subtilités des données issues du terrain (Glaser, 1978 ; Strauss \& Corbin, 1990). Cette notion de «sensible » est difficilement saisissable. Il est possible de distinguer deux courants principaux de signification. « Un premier courant de sens distingue trois niveaux : la capacité de sensation et de perception comme on pourrait le dire du côté de la souffrance, d'un point névralgique; la capacité de vie affective intense qui renvoie à l'émotivité ou qui caractérise les personnes impressionnables ; le fait de se laisser toucher, d'être réceptif (être sensible à...) ou, techniquement, de réagir au contact comme les plaques sensibles ou des appareils de haute précision» (Vulbeau, 2007). Sur le plan de la recherche, ce premier courant est identifié par les Anglo-saxons comme «sensitive research ». Est alors considérée comme sensible toute recherche qui pose potentiellement une menace aux personnes impliquées dans la recherche. D’après Lee (1993), ces menaces sont au nombre de trois. La première est qualifiée de «menace intrusive » et concerne l'ensemble des questions relevant de la sphère privée, du sacré, mais également de ce qui peut être perçu comme particulièrement stressant pour les participants (la sexualité, la mort, la religion...). La deuxième menace est qualifiée de "menace sanction » et fait référence aux recherches susceptibles de révéler des informations stigmatisantes ou incriminantes (déviances, les comportements illicites et illégaux). Le troisième type de menace est politique et fait référence aux recherches qui vont questionner les différents pouvoirs en place. Un second groupe sémantique qualifie de sensible ce qui peut être identifié par les sens. La recherche sensible fait alors référence aux recherches dans lesquelles les sens auront une part centrale dans la production de données de recherche. Ces recherches sont identifiées par les Anglo- 
saxons comme «sensory research ». On assiste aujourd'hui à l'émergence des recherches « sensorielles » (Howes, 2009) qui se traduit par le développement de méthodes visuelles, (Banks, 2007) mais également d'une anthropologie de l’olfaction (Candau \& Jeanjean, 2006). D’autres se prononcent en faveur d'une approche multisensorielle et revendiquent une méthodologie ethnographique sensorielle (Pink, 2009).

Dans la continuité des réflexions engagées sur les terrains sensibles, le numéro 7 de la revue SpécifiCités offrira un espace de discussion et de réflexion sur ce paradigme de la recherche sensible. Les travaux pourront aborder ce paradigme aussi bien d'un point de vue empirique que théorique. Conformément à la politique éditoriale de la revue, nous étudierons avec intérêt tout type de proposition. Nous encouragerons les propositions d'articles consacrés aux dimensions méthodologiques et techniques qui s’imposent au chercheur (accès aux terrains, définition de l'échantillon, analyse des données...). Nous porterons également un intérêt particulier pour les travaux traitant des dimensions relationnelles et éthiques de la recherche sensible (rapports aux participants, confidentialité, consentement...).

\section{Conditions de soumission}

Les articles (30000 signes environ) soumis seront évalués anonymement et rigoureusement par un comité de lecture. Les propositions devront être remises avant le 30 juin 2013 pour une parution du dossier octobre 2013. Elles devront respecter les normes de présentation de la revue disponibles dans le document attaché en pièce jointe. Vous êtes prié(e) d'envoyer une proposition de communication (titre et 300 mots) avant le 15 mai 2013.

Les propositions doivent être adressées coordonnateurs du numéro 7 de la revue :

\section{Olivier Brito}

olivierbrito@gmail.com

Sébastien Pesce

s.pesce@wanadoo.fr 